\title{
Mais vale prevenir que remediar? A dificuldade de decisão
}

Joana Oliveira ${ }^{1}$

\section{RESUMO}

O médico de família tem um papel fulcral na promoção da saúde e prevenção da doença pelo seu contacto mais próximo com o utente, família e comunidade. Este deve incentivar a prática de estilos de vida saudáveis em todos os seus contactos com o utente e diagnosticar precocemente situações de doença, no contexto de sinais e sintomas sugestivos. Para além disso, existem rastreios que podem ser aplicados. Por outro lado, existem cada vez mais dúvidas acerca do benefício de realizar check-ups a toda a população, procurar e tratar incessantemente fatores de risco dos indivíduos, havendo cada vez mais evidência da inexistência de benefícios na morbimortalidade e do crescente risco das intervenções efetuadas.

Palavras-chave: Prevenção; Médico de família; Prevenção quaternária.

\section{INTRODUÇÃO}

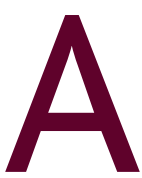

prevenção da doença é uma das pedras basilares do trabalho do médico de família (MF), que o distingue de outras especialidades médicas. AWONCA Europa, com o apoio e colaboração da Organização Mundial da Saúde, em 2002, definiu várias competências nucleares do MF, sendo uma delas a abordagem abrangente, que inclui a "promoção da saúde e do bem-estar através da aplicação adequada de estratégias de promoção da saúde e de prevenção da doença". ${ }^{1}$ Mais do que prevenir a doença, o MF deve promover a saúde, incentivando estilos de vida saudáveis, aproveitando todas as oportunidades de contacto, quer em consultas de rotina quer em consultas de seguimento de patologias crónicas.

No contexto comunitário e de proximidade em que trabalha o MF, a promoção da saúde e a prevenção primária da doença são de mais fácil aplicação e um dos trunfos de que dispõe. A prevenção primária consiste em reduzir a incidência de doença na população, diminuindo os fatores de risco que causam doença. ${ }^{2} \mathrm{En}$ tre o trabalho assistencial de resposta às necessidades dos utentes da sua lista, o MF deve despender do seu tempo para pôr em prática aquilo que o define: preve-

1. USF Manuel Rocha Peixoto. Braga, Portugal. nir! Podem ser realizadas sessões de educação para a saúde em grupos definidos, sempre adaptando os conteúdos e métodos ao público-alvo. E isto é que é ser MF no seu todo, com uma visão holística do utente/família. É certo que este nível de prevenção pode também ser executado em contexto de consulta, com especial destaque na consulta de saúde infantil, saúde da mulher, acompanhamento de diabéticos e hipertensos. A vacinação tem aqui também especial importância, diminuindo a mortalidade infantil e erradicando muitas doenças infeciosas, ${ }^{3}$ devendo por isso ser incentivado o seu cumprimento. Este é um dos maiores orgulhos da medicina geral e familiar, que enaltece o importante papel do trabalho em equipa que aqui se desenvolve: MF, enfermeiro de família e secretário clínico.

O MF tem ainda um papel fundamental na deteção precoce de doenças, através da realização de rastreios. Os rastreios têm como principal objetivo o diagnóstico de doença em pessoas assintomáticas. ${ }^{2} \mathrm{E}$ aqui surgem muitos debates e controvérsias que dividem a classe médica. A desinformação em saúde e a criação de ideias erradas, fomentando o medo na população, são comuns, fazendo com que procurem cuidados médicos muitas vezes motivados pela realização de check-ups completos, crentes que assim assegurarão a manutenção da sua saúde, simultaneamente tranquilizando-os 
ou desculpabilizando-os de possíveis erros nos seus estilos de vida. Podemos estar, através da utilização indiscriminada de análises clínicas ou exames de imagem de rotina, a fomentar práticas pouco saudáveis. É certo que os exames complementares de diagnóstico podem estar normais, mesmo na presença de morbilidade, o que pode fazer com que as pessoas ignorem sintomas de alerta, sobrevalorizando a normalidade dos exames. Assim, poderá existir um efeito reverso do desejado com o pedido exaustivo de exames: uma deteção ainda mais tardia de situações que poderiam ser prevenidas.

Numa revisão da Cochrane, publicada em 2019, é afirmada a inexistência de benefício da realização de check-ups gerais à população geral, associadamente a um aumento de intervenções diagnósticas e terapêuticas, por vezes desproporcionais ao risco clínico, em pessoas assintomáticas. ${ }^{4}$ Existe pouco ou nenhum efeito na mortalidade total, por cancro e, provavelmente, cardiovascular com os check-ups, bem como na diminuição de eventos cardíacos e cerebrais isquémicos fatais e não-fatais. É reforçado o benefício em realizar atividades preventivas e testes clinicamente motivados em situações específicas, nomeadamente no acompanhamento e monitorização de pessoas com elevado risco cardiovascular. O benefício da realização de check-ups universais, muitas vezes fomentado por grupos privados, não é suportado pela melhor evidência científica do momento.

O benefício de testes de rastreio é variável. O rastreio do cancro da próstata não reduz a mortalidade-específica por essa causa, podendo até causar dano ao indivíduo pelo excesso de exames de diagnóstico invasivos e tratamentos desproporcionais ao problema. ${ }^{5} \mathrm{~A}$ pesquisa de sangue oculto nas fezes reduz a mortalidade por cancro colorretal, embora aumente consideravelmente o número de exames invasivos em pessoas saudáveis. ${ }^{6}$ Assim, o MF deve, mediante um acordo com o utente, escolher o que melhor se ajusta em cada caso, evitando a globalização de exames com pouca evidência.

Para além disso, é importante não cair no erro de ceder a tratar fatores de risco, correndo o risco de criar doença. Somos muitas vezes questionados sobre a pressão arterial e pressionados a medicar para obter valores «normais», mesmo quando estamos perante uten- tes de baixo risco cardiovascular com pressão arterial ligeiramente acima do recomendado. Mesmo as recomendações médicas não são coerentes entre si, o que dificulta ainda mais o trabalho do MF. Uma investigação realizada de 1998 a 2015 em adultos (18-74 anos) com hipertensão ligeira (pressão arterial não tratada de 140-159/90-99mmHg), sem antecedentes de doença cerebrocardiovascular, ${ }^{7}$ demonstrou que nestes indivíduos a instituição de terapêutica anti-hipertensiva não diminui a mortalidade nem a doença cardiovascular. Ao invés, o tratamento está associado ao aumento de efeitos laterais, nomeadamente hipotensão, síncope, anomalias eletrolíticas e lesão renal aguda. Existe discrepância entre este estudo e as guidelines, uma vez que as últimas se baseiam em grupos de elevado risco cardiovascular, fazendo recomendações generalizadas para os grupos de baixo risco e, por isso, não podendo ser aplicadas de forma global nos cuidados de saúde.

Muitas vezes o MF tende a exercer medicina defensiva, perdendo-se muitas vezes a essência da verdadeira Medicina, baseada na história clínica e exame físico, ao invés dos exames complementares de diagnóstico (complementares e não essenciais ao diagnóstico!); com foco na educação para a saúde e prevenção da doença e tratamento das situações que assim o justifiquem, ao invés da procura incessante de fatores de risco e tratamento dos mesmos, sem benefício na morbilidade e mortalidade. Nisto se baseia o conceito de prevenção quaternária: identificação dos indivíduos em risco de excesso de tratamentos, protegendo-os de intervenções médicas invasivas, diminuindo os danos causados pela medicina..$^{8-9}$ Deve ser evitada a prática de disease mongering, muitas vezes incentivada pelas agências farmacêuticas e outras instituições privadas. Algumas decisões clínicas são realizadas com alguma incerteza, nomeadamente na prestação de cuidados preventivos e curativos. Enquanto na prestação de cuidados preventivos essa incerteza poderá ser aceitável pelo benefício na saúde do doente, o mesmo não se aplica aos cuidados preventivos, sendo neste último caso imprescindível que os benefícios se sobreponham aos riscos. ${ }^{9}$

Por tudo o que foi exposto, é fácil perceber a dicotomia vivida diariamente pelo MF. Por um lado, o utente que pretende a realização de estudos exaustivos mesmo que assintomático, achando que isso o beneficia; 
por outro, o conhecimento da evidência mais recente por parte do MF que coloca de parte essa prática. Assim, é premente o investimento na literacia em saúde da população, dotando-a de conhecimentos e ferramentas sempre com o objetivo e o foco na melhoria da saúde do indivíduo, das famílias e da comunidade.

\section{AGRADECIMENTOS}

À Dra. Maria João Botelho, pela dedicação e apoio no meu processo de formação. Excelente Médica de Família.

\section{REFERÊNCIAS BIBLIOGRÁFICAS}

1. Allen J, Gay B, Crebolder H, Heyrman J, Svab I, Ram P, et al. A definição europeia de medicina geral e familiar (clínica geral/medicina familiar) [The European definition of family medicine (general practice/family medicine)]. Rev Port Med Geral Fam. 2005;21(5):511-6. Portuguese

2. Downie RS, Tannahill C, Tannahill A. Health promotion, models and values. Oxford: Oxford University Press; 1990. ISBN 9780192619280

3. Direção-Geral da Saúde. Programa nacional de vacinação 2017. Lisboa: DGS; 2016.

4. Krogsbøll LT, Jørgensen KJ, Gøtzsche PC. General health checks in adults for reducing morbidity and mortality from disease. Cochrane Database Syst Rev. 2019;1(1):CD009009.

5. Martin RM, Donovan JL, Turner EL, Metcalfe C, Young GJ, Walsh El, et al. Effect of a low-intensity PSA-based screening interventionon prostate cancer mortality: the CAP randomized clinical trial. JAMA. 2018;319 (9):883-95.

6. Holme $\varnothing$, Bretthauer M, Fretheim A, Odgaard-Jensen J, Hoff G. Flexi- ble sigmoidoscopy versus faecal occult blood testing for colorectal cancer screening in asymptomatic individuals. Cochrane Database Syst Rev. 2013;(9):CD009259.

7. Sheppard JP, Stevens S, Stevens R, Martin U, Mant J, Hobbs FD. Benefits and harms of antihypertensive treatment in low-risk patients with mild hypertension. JAMA Intern Med. 2018;178(12):1626-34.

8. Jamoulle M. Quaternary prevention: prevention as you never heard before (definitions for the four prevention fields as quoted in the WONCA international dictionary for general/family practice). Bruxelles: Groupe de Recherche de Médecine de Famille et Soins Primaires; 2000 [cited 2019 Feb 17].Available from: http://www.ulb.ac.be/esp/mfsp/quaten.html

9. Melo M.A prevenção quaternária contra os excessos da medicina [Quaternary prevention against the excesses of medicine]. Rev Port de Clin Geral. 2007;23(3):289-93. Portuguese

\section{CONFLITO DE INTERESSES}

O autor declara não possuir quaisquer conflitos de interesse.

\section{FINANCIAMENTO DO ESTUDO}

Os autores declaram não ter recebido qualquer financiamento do estudo.

\section{ENDEREÇO PARA CORRESPONDÊNCIA}

Joana Oliveira

E-mail: joanaferreiraoliveira91@gmail.com

https://orcid.org/0000-0002-4549-6754

\section{Recebido em 30-03-2019}

Aceite para publicação em 27-09-2019

\section{ABSTRACT}

\section{BETTER SAFE THAN SORRY? THAT IS THE QUESTION}

The family doctor has a central role in promoting health and preventing disease through his closest contact with the patient, family, and community. This should encourage the practice of healthy lifestyles in all its contacts with the patient and early diagnosis disease situations, in the context of suggestive signs and symptoms. In addition, there are screenings that can be applied. On the other hand, there are more and more doubts about the benefit of performing check-ups to the entire population, constantly seeking, and treating risk factors for individuals, with more and more evidence of the lack of benefits in morbidity and mortality and the growing risk of interventions

Keywords: Prevention; Family doctor; Quaternary prevention. 